\title{
Cytomorphometric analysis of squames from oral premalignant and malignant lesions.
}

\author{
Veda Hegde ${ }^{1}$. \\ ${ }^{1}$ MDS, Reader . Department of Oral Pathology, SDM College of Dental Sciences and Hospital, Dharwad - 580009.
}

Correspondence:

Department of Oral Pathology,

SDM College of Dental Sciences and Hospital,

Dharwad-580009.

Email-hegdeveda6@rediffmail.com

Hegde V. Cytomorphometric analysis of squames from oral premalignant and malignant lesions. J Clin Exp Dent. 2011;3(5):e441-4. http://www.medicinaoral.com/odo/volumenes/v3i5/jcedv3i5p441.pdf

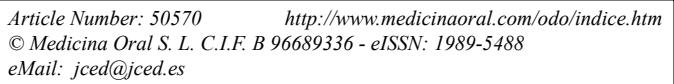

\begin{abstract}
Objective: Oral cytology is becoming increasingly important in the early diagnosis of oral cancer, including epithelial atypia. The aim of this study is to assess the whether the cytoplasmic diameter, nuclear diameter and their nuclear to cytoplasmic ratio are reliable parameters in exfoliative cytology in detecting premalignant and malignant lesions.

Study design: Cell and nuclear diameter were measured from the squames of normal buccal mucosal and of oral leukoplakia, oral submucous fibrosis, oral lichen planus which were histologically benign and oral squamous cell carcinoma were measured using an ocular micrometer disc. The nuclear to cytoplasmic ratio in each of these were deduced from the values obtained and compared with the controls which were age, sex and site matched. The data were evaluated using statistical methods, namely 't' test and analysis of variance.

Results: We obtained a statistically significant reduction in the mean cytoplasmic and nuclear diameter in all the four groups. The nuclear to cytoplasmic ratio revealed to be significant only in group 1,2 and 4 .

Conclusion: The present study suggests that a decrease in the mean cytoplasmic diameter of exfoliated buccal mucosal cells could serve as an early indicator of dysplastic change especially in lesions which appear histologically benign.
\end{abstract}

Key Words: Cytomorphometric analysis, exfoliative cytology, premalignant lesions, cancer, dysplasia, quantitative analysis. 


\section{Introduction}

Oral cancer, the most common cancer accounts for almost $40 \%$ of all cancers in the Indian subcontinent (1). Oral malignancy occur twice as many men as women and $95 \%$ are found in persons older than 40 years (2). A significant proportion of squamous cell carcinoma develops from premalignant lesion. Early detection of a premalignant lesion increases the survival and morbidity of patients suffering from these conditions (1).

Histological examination of tissue remains the gold standard for diagnosis and identification of oral lesions ${ }^{1}$. It has been suggested that oral cytology may be useful in detection of early carcinoma or premalignant lesions in asymptomatic patients with innocent appearing lesions (3). Lately oral exfoliative cytology has lately reemerged due to improved methods and application in oral precancer and cancer (1). Quantitative parameters such as cell size, nuclear size, nuclear to cytoplasmic ratio have shown measurable changes in cells obtained from premalignant and malignant lesions (4). Quantitative methods of smear assessment have been shown to enhance the diagnostic reliability of oral exfoliative cytology (5).

We conducted this study to assess the morphometric changes of cell and nuclear diameter and its ratio in exfoliated buccal mucosal cells of oral leukoplakia, oral submucous fibrosis, oral lichen planus and oral squamous cell carcinoma and compare with that of the controls.

\section{Material and Methods}

Patient selection

Patients visiting our institution constituted both the stu- dy and control groups. Ten cases for each of the study groups (ten cases of oral leukoplakia as study group and ten cases of control group, ten cases of oral submucous fibrosis and ten cases of control group, ten cases of oral lichen planus and ten cases of study group, ten cases of oral squamous cell carcinoma and ten cases of study group) along with ten cases each of control groups for each of the study groups constituted our study population. Histologically diagnosed cases of oral leukoplakia, oral submucous fibrosis, and oral lichen planus that were histologically benign and oral squamous cell carcinoma of the buccal mucosa constituted the study group. These were categorized as group 1, 2, 3 and 4 respectively. The control groups consisted of subjects who did not have any lesions in the area of the buccal mucosa and were age and sex matched with the study group.

Smear preparation and staining

We obtained the buccal mucosal smears from both the study and control groups using a sterile wooden spatula. The smear was fixed for a minimum period of fifteen minutes in $95 \%$ ethyl alcohol and stained using the papaniculaou method.

\section{Morphometric analysis}

We measured the cell diameter and nuclear diameter with the help of a calibrated eye piece graticule in $100 \mathrm{X}$ objective. We achieved this by superimposing the eyepiece graticule on the stained cells. We measured only clearly defined cells avoiding clumped and folded cells. In each slide hundred cells were measured in both the horizontal and vertical axis and an average of the two was taken. We deduced the nuclear to cytoplasmic ratio from the above values.

\begin{tabular}{|c|c|c|c|c|c|}
\hline & & Mean & Standard Deviation & $\mathrm{t}$ & $\mathrm{P}$ \\
\hline \multirow{2}{*}{ Cell Diameter } & Leukoplakia & 29.7090 & 5.7325 & \multirow{2}{*}{9.741} & \multirow{2}{*}{0.000} \\
\hline & Normal & 49.0290 & 2.5446 & & \\
\hline \multirow{2}{*}{ Nuclear Diameter } & Leukoplakia & 6.3170 & 0.5134 & \multirow{2}{*}{8.094} & \multirow{2}{*}{0.000} \\
\hline & Normal & 8.0090 & 0.4164 & & \\
\hline \multirow{2}{*}{ Nuclear / cytoplasmic ratio } & Leukoplakia & 0.21700 & 0.03197 & \multirow{2}{*}{5.130} & \multirow{2}{*}{0.000} \\
\hline & Normal & 0.1635 & 0.47019 & & \\
\hline
\end{tabular}

Table 1. Comparison of mean values of cells diameter, nuclear diameter and nuclear to the cytoplasmic ratio in leukoplakia and normal squames of the buccal mucosa.

\begin{tabular}{|c|c|c|c|c|c|}
\hline & & Mean & Standard Deviation & $\mathrm{t}$ & $\mathrm{P}$ \\
\hline \multirow{2}{*}{ Cell Diameter } & Oral submucous fibrosis & 37.1140 & 6.8309 & \multirow{2}{*}{4.631} & \multirow{2}{*}{0.000} \\
\hline & Normal & 47.6950 & 2.5333 & & \\
\hline \multirow{2}{*}{ Nuclear Diameter } & Oral submucous fibrosis & 7.0830 & 0.4287 & \multirow{2}{*}{4.353} & \multirow{2}{*}{0.000} \\
\hline & Normal & 8.0700 & 0.5747 & & \\
\hline \multirow{2}{*}{ Nuclear / cytoplasmic ratio } & Oral submucous fibrosis & 0.19550 & 0.3659 & \multirow{2}{*}{2.183} & \multirow{2}{*}{0.043} \\
\hline & Normal & 0.1689 & 0.12693 & & \\
\hline
\end{tabular}

Table 2. Comparison of mean values of cell diameter and nuclear diameter and nuclear to cytoplasmic ration in oral submucous fibrosis and normal squames of the buccal mucosa. 


\section{Statistical Analysis}

We used the t' test to identify the differences in the mean values of cell diameter and nuclear diameter between the study and control in each of the four groups and Analysis of Variance to evaluate the differences in cell diameter and nuclear diameter and the nuclear to cytoplasmic ratio between the different groups. The data were collected on forms and entered into a Microsoft Excel Worksheet and analyzed using SPSS (ver.7.5) statistical package.

\section{Results}

We obtained a significant reduction in the mean cytoplasmic and nuclear diameter in all the four groups when compared to their controls .There was a significant increase in the mean values of the nuclear to cytoplasmic ratio only in group 1, 2 and 4. There was no evidence of dysplastic features seen histopathologically in group 1 , 2 , and 3 (Table $1-4$ ).

\section{Discussion}

The incidence of oral precancer and cancer is high in India. Several large scale and long term epidemiological studies have correlated the relationship between precancer and cancer. Quantitative cytomorphometric assessment of exfoliated buccal mucosal cells has shown measurable changes in cells obtained from malignant and premalignant lesions. Moreover exfoliative cytology offers a simple non-invasive technique that can be repeated frequently with little discomfort to the patient (4).

Exfoliative cytology has always been a subject of contro- versy for detecting premalignant and malignant lesions. Cytologic smears were found to be useful in lesions of leukoplakia and oral submucous fibrosis (6). It has been also been useful for diagnosing very early malignant change $(3,7,8)$. A high degree of correlation was obtained between smear and biopsy in an experimentally induced tumour in the hamster cheek pouch. It was also demonstrated that exfoliative accurately reflects early epithelial dysplasia in the development of the experimental tumour (9).

With reference to the cytoplasmic changes observed in the exfoliated cells, Ogden et al obtained a reduction in the cytoplasmic area of normal buccal mucosal cells in patients with malignant disease distant from the oral cavity (10). Ogden et al studied field change in oral cancer using oral exfoliative cytology and found that there was a decrease only in the cytoplasmic area in patients with and without the habit of smoking and alcohol intake (11). Cowpe et al concluded that a reduction in cell area remains an early indication of dysplastic change and that tissues undergoing malignant transformation typically show a reduction in the cell areas before reduction in the nuclear area (12). In our study, there was a significant reduction in the cytoplasmic diameters in all the four groups when compared to their controls. In case of group 1,2, and 3, the reduction was evident in exfoliated cells even without histologic evidence of dysplasia (Table 1-4).

Ramaesh et al obtained greatest reduction in the mean cytoplasmic area of squames in squamous cell carcinoma and leukoplakia with histologic evidence of dyspla-

\begin{tabular}{|c|c|c|c|c|c|}
\hline & & Mean & Standard Deviation & $\mathrm{t}$ & $\mathrm{P}$ \\
\hline \multirow{2}{*}{ Cell Diameter } & Lichen Planus & 37.3740 & 5.7987 & \multirow{2}{*}{5.667} & \multirow{2}{*}{0.000} \\
\hline & Normal & 48.571 & 13.6382 & & \\
\hline \multirow{2}{*}{ Nuclear Diameter } & Lichen Planus & 6.8380 & 0.4743 & \multirow{2}{*}{6.826} & \multirow{2}{*}{0.000} \\
\hline & Normal & 8.8380 & 0.4743 & & \\
\hline \multirow{2}{*}{ Nuclear / cytoplasmic ratio } & Lichen Planus & 0.18660 & 0.3285 & \multirow{2}{*}{1.948} & \multirow{2}{*}{0.067} \\
\hline & Normal & 0.1661 & 0.44685 & & \\
\hline
\end{tabular}

Table 3. Comparison of mean values of cell diameter and nuclear diameter and nuclear to the cytoplasmic ratio in oral lichen planus and normal squames of the buccal mucosa.

\begin{tabular}{|c|c|c|c|c|c|}
\hline & & Mean & Standard Deviation & $\mathrm{t}$ & $\mathrm{P}$ \\
\hline \multirow{2}{*}{ Cell Diameter } & Oral squamous cell carcinoma & 28.1240 & 4.4917 & \multirow{2}{*}{1.30414} & \multirow{2}{*}{0.00} \\
\hline & Normal & 48.8070 & 1.8974 & & \\
\hline Nuclear Diameter & $\begin{array}{l}\text { Oral squamous cell } \\
\text { carcinoma } \\
\text { Normal }\end{array}$ & $\begin{array}{l}6.9890 \\
7.9720 \\
\end{array}$ & $\begin{array}{l}0.6322 \\
0.2858 \\
\end{array}$ & 4.480 & 0.00 \\
\hline Nuclear / cytoplasmic ratio & $\begin{array}{l}\text { Oral squamous cell } \\
\text { carcinoma } \\
\text { Normal }\end{array}$ & $\begin{array}{l}0.2500 \\
0.16290\end{array}$ & $\begin{array}{l}0.03615 \\
0.00412\end{array}$ & 7.569 & 0.00 \\
\hline
\end{tabular}

Table 4. Comparison of mean values of cell diameter and nuclear diameter and nuclear to the cytoplasmic ratio in oral squamous cell carcinoma and normal squames of the buccal mucosa. 
sia but no reduction was seen in those cells where histologically there was no evidence of dysplasia (4).

A similar reduction was observed in the nuclear diameter in all the four groups when compared to their controls (Table 1-4). Ramaesh et al found an increase in the mean nuclear diameter of exfoliated cells of leukoplakia with histologic evidence of dysplasia whereas no change in nucleus was seen morphometrically in leukoplakia without histologic evidence of dysplasia $(4,13)$. Cowpe et al found nuclear size to vary with advancing age in normal oral mucosal squames and did not obtain an increase in abnormal lesions. He thus concluded that an increase in nuclear size is not always a clear feature for early cytologic indication dysplastic change (12).

When nuclear to cytoplasmic ratio was assessed, an increase in the nuclear to cytoplasmic ratio was observed in group 1, 2, and 4 (Table 1-4). An increased nuclear to cytoplasmic ratio is a known feature in squamous cell carcinoma. We still considered this in our study along with normal buccal mucosal cells to serve as a baseline our cases of oral leukoplakia, and oral submucous fibrosis and oral lichen planus which appeared histologically benign. Callimeri and Smith found that an increased nuclear to cytoplasmic ratio was one of the consistent findings during progression from benign to a state of malignancy (14). Cowpe found that in malignant lesions, an increased nuclear to cytoplasmic ratio was due to a reduction in the mean cytoplasmic area and an elevation in the mean nuclear area; while in case of epithelial dysplasia, an increased nuclear to cytoplasmic ratio was only due to a significant reduction in the mean cell area with little or no change in the mean nuclear area (15). While our cases appeared histologically very benign, morphometric analysis showed consistent finding of dysplastic change in relation to increased nuclear to cytoplasmic ratio. Therefore even before nuclear changes are evident, only cytoplasmic changes could serve as an important and early parameter for dysplastic change in the exfoliated cells. Moreover, exfoliative cytology being a simple, non- invasive technique can be repeated with least discomfort to the patient.

Thus, to conclude cytoplasmic changes alone without any nuclear change could serve as an important and early parameter in exfoliated cells especially when there is no histologic evidence of dysplasia. As exfoliative cytology is a simple, non-invasive technique, cytomorphometric analysis of exfoliated cells should be done for regular follow up of premalignant lesions. It is also very useful in those cases where biopsy is contraindicated; and its cost effectiveness and compliance for this technique makes this procedure useful for mass screening and regular follow up of patients with suspicious lesions where biopsy is contraindicated.

\section{References}

1. Mehrotra R, Gupta A, Singh M, Ibrahim R. Application of cytology and molecular biology in diagnosing premalignant or malignant oral lesions. Mol Cancer. 2006; 5:11.

2. Silverman S Jr. Early diagnosis of oral cancer. Cancer. 1988; 62; 1796-9.

3. Umiker WO, Lampe I, Rapp R, Hiniker JJ. Oral smears in the diagnosis of carcinoma and premalignant lesion. Oral Surg Oral Med Oral Pathol. 1960; 13: 897-907.

4. Ramaesh T, Mendis BR, Ratnatunga N, Thattil RO. Cytomorphometric analysis of squames obtained from normal oral mucosa and lesions of oral leukoplakia and squamous cell carcinoma. J Oral Pathol Med. 1998; 7: 83-6.

5. Sugarman PB, Savage NW. Exfoliative cytology in clinical oral pathology. Aust dental J. 1996; 41(2): 71-4.

6. Peters H. Cytologic smears from the mouth: Cellular changes in disease and after radiation. Am J Clin Pathol. 1958; 29: 219-25.

7. Alling CC, Secord RT. A technique for oral exfoliative cytology. Oral Surg Oral Med Oral Pathol. 1964; 17: 669-76.

8. Shapiro BL, Gorlin RJ, Jordan WA. The role exfoliative cytology in oral cancer detection. Oral Surg Oral Med Oral Pathol. 1964; 17: $327-30$

9. Stahl SS. Correlation of cytodiagnosis and biopsy in the evolution of an experimentally induces carcinoma. Oral Surg Oral Med Oral Pathol. 1963; 16: 985-93

10. Ogden GR, Cowpe JG, Wright AJ. Oral exfoliative cytology: review of methods of assessment. J Oral Pathol Med. 1997; 26: 201-25.

11. Ogden GR, Cowpe JG, Green NW. Detection of field change in oral cancer using oral exfoliative cytologic study. Cancer. 1991; 68: $1611-5$

12. Cowpe JG, Longmore RB, Green NW. Quantitative exfoliative cytology of abnormal oral mucosal smears. J Roy Soc Med. 1988; 81: 509- 13.

13. Ramaesh T, Mendis BR, Ratnatunga N, Thattil RO. The effect of tobacco smoking and of betel chewing with tobacco on the buccal mucosa: a cytomorphometric analysis. J Oral Pathol Med. 1999; 28: $385-88$.

14. Callimeri GE, Smith CJ. Exfoliative cytology of early lesions of experimental oral cancer in the hamster, Arch Oral Biol. 1965; 10: 465-70.

15. Cowpe JG. Quantitative exfoliative cytology of normal and abnormal oral mucosal squames: preliminary communication. J Roy Soc Med. 1984; 77: 928-31. 